\title{
Sufficient Conditions for the Instability of Numerical Integration Methods*
}

\author{
Abbas I. Abdel Karim**
}

(March 5, 1969)

\begin{abstract}
In a previous paper, a general theorem was investigated for the stability of numerical integration methods for the solution of systems of differential equations. In this paper, further theorems are developed as sufficient conditions for the instability of numerical integration methods. Applying these theorems, the instability of known formulas are checked easily at a glance.
\end{abstract}

Key words: Numerical integration; stability.

The system of $m$ ordinary differential equations can be written in the vector form as follows:

$$
Y^{\prime} \equiv \frac{d Y}{d x}=F(x ; Y)
$$

with the initial value $Y(a)=Y_{0}, x \epsilon[a, b]$.

Let the vectors:

(i) $Y_{n}$ be the exact solution of the following difference equation at $x=x_{n}$ :

$$
Y_{n+1}=\sum_{\nu=-q}^{0} a_{\nu} Y_{n+\nu}+h \sum_{\nu=-q}^{1} b_{\nu} Y_{n+\nu}^{\prime}
$$

(ii) $\stackrel{*}{Y}_{n}$ be the (practical) computed solution of (1) at $x=x_{n}$ according to:

$$
\stackrel{*}{Y}_{n+1}=\sum_{\nu=-q}^{0} a_{\nu} \stackrel{*}{Y+\nu}_{n+h} \sum_{\nu=-q}^{1} b_{\nu} F\left(x_{n+\nu} ; \stackrel{*}{Y}_{n+\nu}\right)+R_{n+1},
$$

where $R_{n+1}$ is the round off error.

(iii) $Y\left(x_{n}\right)$ be the exact solution of (1) at $x=x_{n}$. Thus the corresponding exact formula is:

$$
Y\left(x_{n}+h\right)=\sum_{\nu=-q}^{0} a_{\nu} Y\left(x_{n}+\nu h\right)+h \sum_{\nu=-q}^{1} b_{\nu} F\left(x_{n}+\nu h ; Y\left(x_{n}+\nu h\right)\right)+T_{k+1} .
$$

The truncation error $T_{k+1}=\mu_{k+1} h_{\leftarrow}^{k+1} Y_{\left(x_{n}\right)}^{(k+1)}+0\left(h^{k+2}\right), \quad k \geqslant 1, \quad \mu_{k+1} \neq 0$.

(iv) $\epsilon_{n}$ be the error of the solution at the point $x_{n}$, thus:

$$
\epsilon_{n}=\stackrel{*}{Y}_{n}-Y\left(x_{n}\right)
$$


In Karim's paper [1], ${ }^{1}$ we discussed the following:

1. Corresponding to the integration formula (2), there is a nonhomogeneous system of linear difference equations with constant coefficients for the error $\epsilon_{n}$ in the form:

$$
\epsilon_{n+1}=\sum_{\nu=-q}^{0} a_{\nu} \epsilon_{n+\nu}+h \tilde{J} \sum_{\nu=-q}^{1} b_{\nu} \epsilon_{n+\nu}+\tilde{R}, \quad \tilde{R} \neq 0
$$

Where, in a small interval $\left(x_{-q}, x_{1}\right): \tilde{J}=\left(\frac{\partial f_{i}}{\partial y_{j}}\right)$ is a constant matrix $(i, j=1(1) m)$ and $\tilde{R}=R_{n+1}-T_{k+1}$ is a nonzero constant vector, see Hamming (1959) [2].

2. Corresponding to the system of difference equations (6) there is a system of the characteristic polynomial equations:

$$
P\left(\lambda_{i}\right) \equiv \lambda_{i}-\sum_{\nu=-q}^{0} a_{\nu} \lambda_{i}^{\nu}-h \rho_{i} \sum_{\nu=-q}^{1} b_{\nu} \lambda_{i}^{\nu}=0, \quad i=1(1) m
$$

where: $\rho_{i}=$ the eigenvalues of the matrix $\tilde{J}$.

3. The numerical integration method (2) is said to be stable according to Wilf's criterion (1959) [3] if the roots of (7) for all $i=1(1) \mathrm{m}$ are inside the unit circle in the complex plane, so that the error introduced at any stage tends to decay than build up. Expanding the terms of (4) according to Taylor, and comparing the coefficients of $h^{j}$, we get the following system of linear equations:

$$
1-j b_{1}=\sum_{\nu=-q}^{-1} \frac{j}{\nu}\left(a_{\nu}+\frac{j}{\nu} b_{\nu}\right)+\gamma_{1} a_{0}+\gamma_{2} b_{0}+\gamma_{3}(k+1) ! \mu_{k+1}, \quad j=0(1) k+1
$$

where:

$$
\gamma_{1}=\left\{\begin{array}{l}
1 \\
0
\end{array} \text { for } \begin{array}{l}
j=0 \\
j \neq 0
\end{array}, \quad \gamma_{2}=\left\{\begin{array}{l}
1 \\
0
\end{array} \text { for } \begin{array}{r}
j=1 \\
j \neq 1
\end{array}, \quad \gamma_{3}=\left\{\begin{array}{l}
1 \\
0
\end{array} \text { for } \begin{array}{l}
j=k+1 \\
j \neq k+1
\end{array} .\right.\right.\right.
$$

For $j=0$ and $j=1$ it follows from (8):

and

$$
\sum_{\nu=-q}^{0} a_{\nu}=1
$$

$$
\sum_{\nu=-q}^{0} \nu \cdot a_{\nu}+\sum_{\nu=-q}^{1} b_{\nu}=1
$$

REMARK: Many incorrect formulas of the form (2) are found in some references. It is necessary to check such cases at least by formulas (9) and (10). In many cases it is sufficient to use (9) and (10) for the corrections. especially in case of a sign mistake or in case of some wrong coefficients, otherwise it is necessary to make use of $(8)$ for $j=2,3 \ldots, k+1$. It is possible for example, by using (9) and (10) to recognize immediately that eleven of the eighteen formulas, due to H. J. Gray (1955) [4] (tables 4, page 14, formulas Nr. 6, 9, $10 \ldots$., 18) have thirteen mistakes. Using (9) and (10), the mistakes can be corrected. Also two formulas by W. Quade (1957) [5] (p. 160 formula (6.2') and p. 167 formula (9.4)) have written mistakes.

THEOREM (1): The integration formula (2) for the solution of $\mathrm{m}$ ordinary differential equations (1) is stable if and only if, the Hermitian forms with the coefficients

$$
\mathrm{A}_{\mathrm{rs}}^{(\mathrm{i})}=\sum_{\mathrm{l}=0}^{\min (\mathrm{r}, \mathrm{s})}\left\{\overline{\mathrm{C}}_{\mathrm{q}+1+1-\mathrm{r}, \mathrm{i}} \mathrm{C}_{\mathrm{q}+1+1-\mathrm{s} \cdot \mathrm{i}}-\mathrm{C}_{\mathrm{r}-1, \mathrm{i}} \overline{\mathrm{C}}_{\mathrm{s}-1, \mathrm{i}}\right\},
$$

\footnotetext{
${ }^{1}$ Figures in brackets indicate the literature references at the end of this paper.
} 
for all $\mathrm{i}=1,2, \ldots, \mathrm{m}$ are positive definite

where:

$$
\begin{gathered}
\mathrm{C}_{\sigma, \mathrm{i}}=-\left(\mathrm{h} \rho_{\mathrm{i}} \mathrm{b}_{\sigma-\mathrm{q}}+\mathrm{a}_{\sigma-\mathrm{q}}\right), \quad \sigma=0(1)_{\mathrm{q}}+1, \quad \mathrm{a}_{1}=-1, \\
\mathrm{~b}_{1}\left\{\begin{array}{l}
=0 \\
\neq 0
\end{array} \text { if }(2) \text { is } \begin{array}{l}
\text { an extrapolation formula, } \\
\text { an interpolation formula. }
\end{array}\right.
\end{gathered}
$$

The proof of this theorem is to be found in Karim [1].

THEOREM (2): If $\mathrm{a}_{-\mathrm{q}}= \pm 1$ and $\mathrm{b}_{-\mathrm{q}}=-\mathrm{a}_{-\mathrm{q}} \mathrm{b}_{1}$ then the integration formula $(2)$ is unstable.

Proof: Applying theorem (1), the first determinant becomes:

$$
\begin{aligned}
D_{1}^{(i)} & =A_{00}^{(i)}=\left|C_{q+1 . i}\right|^{2}-\left|C_{0, i}\right|^{2} \\
& =\left|h \rho_{i} b_{1}+a_{1}\right|^{2}-\left|h \rho_{i} b_{-q}+a_{-q}\right|^{2} \\
& =\left|h \rho_{i} b_{1}-1\right|^{2}-\left(a_{-q}\right)^{2}\left|h \rho_{i} b_{1}-1\right|^{2}=0 .
\end{aligned}
$$

Thus, it is sufficient that, the integration formula (2) is unstable.

THEOREM (3): If $\sum_{\nu=-\mathrm{q}}^{1}(-1)^{\nu} \mathrm{b}_{\nu}=0$ and $\sum_{\nu=0}^{(\mathrm{q}-\mathrm{s}) / 2} \mathrm{a}_{-\mathrm{q}+2 \nu}=\mathrm{s}=\left\{\begin{array}{l}0 \\ 1 \\ 1\end{array}\right.$ if $\mathrm{q}$ is $\begin{array}{l}\text { even } \\ \text { odd }\end{array}$.

then the integration formula (2) is unstable.

Proof:

1. If $q$ is an even number, we get

$$
\left\{\sum_{\nu=0}^{q / 2} a_{q+2 \nu}+\sum_{\nu=1}^{q / 2} a_{-q+2 \nu} 1\right\}+\left\{\sum_{\nu=0}^{q / 2} a_{-q+2 \nu}-\sum_{\nu=1}^{q / 2} a_{-q+2 \nu-1}\right\}=0 .
$$

Applying (9) it follows:

$$
\sum_{\nu=-q}^{0}(-1)^{v} a_{\nu}=-1
$$

I sing the first assumption of the theorem we get:

$$
\sum_{\nu=-q}^{0}(-1)^{\nu} a_{\nu}+h \rho_{i} \sum_{\nu=-q}^{1}(-1)^{\nu} b_{\nu}=-1
$$

2. If $q$ is an odd number, we get:

$$
\left\{\sum_{\nu=0}^{(q-1) / 2} a_{-q+2 \nu}+\sum_{\nu=1}^{(q-1) / 2} a_{-q+2 \nu-1}\right\}+\left\{\sum_{\nu=0}^{(q-1) / 2} a_{-q+2 \nu}-\sum_{\nu=1}^{(q+1) / 2} a_{-q+2 \nu-1}\right\}=2
$$

Also

$$
\sum_{\nu=1}^{(q+1) / 2} a_{-q+2 \nu-1}-\sum_{\nu=0}^{(q-1) / 2} a_{-q+2 \nu}=-1
$$

this means $\sum_{\nu=-q}^{0}(-1)^{\nu} a_{\nu}=-1$.

Applying the first assumption of the theorem we get:

$$
\sum_{\nu=-q}^{0}(-1)^{\nu} a_{v}+h p_{i} \sum_{\nu=-q}^{1}(-1)^{\nu} b_{\nu}=-1
$$


According to (12) and (12'), $\lambda_{i}=-1$ is a characteristic root of the equation (7), whether $q$ is an odd or even number. Thus, due to Wilf's definition, the integration formula (2) is unstable.

THEOREM (4): If $\sum_{\nu=-\mathrm{q}}^{1} \mathrm{~b}_{\nu}=0$,

then:

(i) The system of the nonhomogeneous difference equations (6) has a nonconstant particular solution.

(ii) The integration method (2) is unstable.

Proof:

(i) To find a particular solution of $(6)$, let

$$
\epsilon_{\nu}=\epsilon=\text { a nonzero constant vector, } \quad \nu=-q(1) 1 \text {. }
$$

Thus we get from (6):

Using (9) and (13) it follows:

$$
\left(1-\sum_{\nu=-q}^{0} a_{\nu}\right) \epsilon=h \tilde{J} \epsilon \sum_{\nu=-q}^{1} b_{\nu}+\tilde{R}
$$

$$
\tilde{R}=0 \text {. }
$$

This means that the assumption (14) contradicts (15) with reference to (6). Put $\epsilon_{\nu}=\nu \epsilon$ in (6), where $\epsilon$ is a nonzero constant vector. Using (9) and (13) we get:

$$
\left(1-\sum_{\nu=-q}^{0} a_{\nu} \cdot \nu\right) \epsilon=h \tilde{J} \sum_{\nu=-q}^{1} b_{\nu} \cdot \nu+\tilde{R}
$$

Hence assuming that $\sum_{\nu=-q}^{1} b_{\nu} \cdot \nu \neq 0$, det $\widetilde{J} \neq 0$, and applying (10) and (13), it can be proved that:

$$
\epsilon=-\left(h \sum_{\nu=-q}^{1} b_{\nu} \cdot \nu\right)^{-1} \tilde{J}^{-1} \tilde{R}
$$

Thus the equations system (6) has a particular solution which is equal to

$$
\epsilon_{n}=-n\left(h \sum_{\nu=-q}^{1} b_{\nu} \cdot \nu\right)^{-1} \tilde{J}^{-1} \tilde{R}
$$

In the case of $\sum_{\nu=-q}^{1} b_{\nu} \cdot \nu=0$, then $\epsilon_{n}$ is a polynomial in $n$ of degree greater than 1 .

(ii) Using (9) and (13) we have:

$$
1-\sum_{\nu=-q}^{0} a_{\nu}-h \rho_{i} \sum_{\nu=-q}^{1} b_{\nu}=0 .
$$

This means $\lambda_{i}=1$ is a characteristic root of the eq (7). Thus, and in addition of (i), the stability condition is not satisfied and the integration formula (2) is unstable.

\section{Illustrative Examples}

The instability of the following integration formulas may be easily checked at a glance:

(1) $Y_{n+1}=Y_{n-1}+2 h Y_{n}^{\prime}$,

$$
T_{3}=\frac{1}{3} h^{3} Y^{\prime \prime \prime}\left(x_{n}\right)+0\left(h^{4}\right)
$$


(2) $Y_{n+1}=-Y_{n-2}+Y_{n-1}+Y_{n}+h\left(-Y_{n-2}^{\prime}+Y_{n}^{\prime}\right)$,

(3) $Y_{n+1}=-Y_{n-2}+Y_{n-1}+Y_{n}+2 h\left(-Y_{n-1}^{\prime}+Y_{n}^{\prime}\right)$,

(4) $Y_{n+1}=-Y_{n-1}+2 Y_{n}+\frac{h}{2}\left(-Y_{n-1}^{\prime}+Y_{n+1}^{\prime}\right)$,

(5) $Y_{n+1}=Y_{n-3}+\frac{4 h}{3}\left(2 Y_{n-2}^{\prime}-Y_{n-1}^{\prime}+2 Y_{n}^{\prime}\right)$,

(6) $Y_{n+1}=Y_{n-3}+Y_{n-2}-Y_{n}+3 h\left(Y_{n-2}^{\prime}+Y_{n}^{\prime}\right)$,

(7) $Y_{n+1}=Y_{n-2}+9 Y_{n-1}-9 Y_{n}+6 h\left(Y_{n-1}^{\prime}+Y_{n}^{\prime}\right)$,

(8) $Y_{n+1}=-Y_{n-2}+Y_{n-1}+Y_{n}+\frac{h}{3}\left(-Y_{n-2}^{\prime}-3 Y_{n-1}^{\prime}+3 Y_{n}^{\prime}+Y_{n+1}^{\prime}\right), T_{6}=-\frac{1}{90} h^{6} Y^{\mathrm{VI}}\left(x_{n}\right)+0\left(h^{7}\right)$

(9) $Y_{n+1}=Y_{n-5}+\frac{3}{10} h\left(11 Y_{n-4}^{\prime}-14 Y_{n-3}^{\prime}+26 Y_{n-2}^{\prime}\right.$ $\left.-14 Y_{n-1}^{\prime}+11 Y_{n}^{\prime}\right)$,

(10)
$T_{3}=h^{3} Y^{\prime \prime \prime}\left(x_{n}\right)+0\left(h^{4}\right)$

$$
T_{4}=\frac{1}{3} h^{4} Y^{\mathrm{IV}}\left(x_{n}\right)+0\left(h^{5}\right)
$$

$T_{4}=-\frac{1}{12} h^{4} Y^{\mathrm{VV}}\left(x_{n}\right)+0\left(h^{5}\right)$

$$
T_{5}=\frac{14}{45} h^{5} Y^{\mathrm{v}}\left(x_{n}\right)+0\left(h^{6}\right)
$$

$T_{5}=\frac{3}{10} h^{5} Y^{\mathrm{v}}\left(x_{n}\right)+0\left(h^{6}\right)$

$T_{5}=\frac{1}{10} h^{5} Y^{\mathrm{V}}\left(x_{n}\right)+0\left(h^{6}\right)$
$T_{8}=-\frac{75}{448} h^{8} Y^{\mathrm{VIII}}\left(x_{n}\right)+0\left(h^{9}\right)$.

$$
+\frac{15}{368} h\left(-8 Y_{n-5}^{\prime}-27 Y_{n-4}^{\prime}+27 Y_{n}^{\prime}+8 Y_{n+1}^{\prime}\right),
$$

According to theorem (2), the formulas (1), (5), (6), (7), and (9) are unstable. According to theorem (4), the formulas (2), (3), (4), (8), and (10) are unstable. Moreover, according to theorem (3), the formula (2) is unstable. Thus all the given formulas (1), (2), . ., (10) are unstable.

Note: The formulas (1), (4), (6), (8), and (10) are given by Quade (1957) [5], (5) is given by Milne (1949) [6], (3) is given by Hamming (1959) [2], (9) is given by Wilf (1960) [7], (2) and (7) are given by the author.

\section{References}

[1] Karim, A. I. A., Criterion for the stability of numerical integration methods for the solution of systems of differential equations, J. Res. NBS, 71 B, (Math. and math. Phys.) Nos. 2 and 3, 91-103 (Apr.-Sept. 1967)

[2] Hamming, R. W., Stable predictor-corrector methods for ordinary differential equations, J. Assoc. Comp. Mach. V. 6, Nr. 1, p. 37-47 (Jan. 1959).

[3] Wilf, H. S., A stability criterion for numerical integration, J. Assoc. Comp. Mach., V. 6, Nr. 3, p. 363-365, July 1959.

[4] Gray, H. J., Jr., Propagation of truncation errors in the numerical solutions of ordinary differential equations by repeated closures, J. Assoc. Comp. Mach. V. 2, Nr. 1, p. 5-17 (Jan. 1955).

[5] Quade, W., Über numerische Integration von gewöhnl. Differential-gleichungen durch Interpolation nach Hermite, ZAMM 37/1957, S. 161-169.

[6] Milne, W. E., Numerical Calculus, chap. V, Princeton, New Jersey 1949.

[7] Wilf, Ralston, Mathematical Methods for Digital Computers, Numerical Integration Methods for the Solution of Ordinary Differential Equations, Part 11, New York, 1960.

(Paper 73B2-291) 